\title{
REACTIVE OXYGEN SPECIES (ROS): A REVIEW
}

\author{
Dr. RamRattan Gupta ${ }^{1}$ Dr. Manu Gupta ${ }^{2}$ Dr. Shivani Bhickta ${ }^{3}$ \\ ${ }^{1}$ Reader, Department of Periodontology, Bhojia Dental College College \& Hospital, Distt. Solan, Himachal Pradesh (India) \\ ${ }^{2}$ Private Practitioner, Punjab (India) \\ ${ }^{3}$ PG Student Department of Periodontology, Bhojia Dental College College \& Hospital, Distt. Solan, Himachal Pradesh (India) \\ Corresponding Author: \\ ${ }^{1}$ Mobile: 919872810521 Email: drgupta116@gmail.com
}

\begin{tabular}{|l|}
\hline Received : \\
$19^{\text {th }}$ August, 2013 \\
Accepted: \\
$22^{\text {nd }}$ Nov, 2013 \\
Available online: \\
$28^{\text {th }}$ Dec, 2013 \\
\hline
\end{tabular}

\section{INTRODUCTION}

In a pioneering paper in 1954, Dan, Rebecca Gerschman and their colleagues related the toxic effects of elevated oxygen levels on aerobes to those of ionizing radiation, and proposed that oxygen toxicity is due to free radical formation. This concept did not capture the imagination of most life scientists, however, until the discovery of the superoxide dismutase enzymes by McCord and Fridovich in 1968. If free radicals play important roles in disease pathologies, then superoxide dismutase (SOD) might have, remarkable properties as a drug for human medicine. The oxidative killing mechanism of neutrophils and other phagocytes involves the formation of reactive oxygen species (ROS). ${ }^{1}$

Free radicals are a family of highly reactive and diverse species, capable of extracting electrons and thereby oxidizing a variety of bio molecules vital to cell and tissue function, which not only includes oxygen free radicals, but also nitrogen and chlorine species. ${ }^{2}$ Mitochondria are still the major source of accidental free radical production. ${ }^{3}$ By definition a free radical is any atom (e.g. oxygen, nitrogen) with at least one unpaired electron in the outermost shell, which is capable of independent existence. A free radical is easily formed when a covalent bond between entities is broken and one electron remains with each newly formed atom ${ }^{4}$. Free radical species are highly reactive and unstable, only becoming

\section{ABSTRACT}

stables by acquiring electrons from, lipids, proteins, nucleic acids, carbohydrates or any nearby molecule and thus causing a cascade of damage and disease. There are two key types of free radical species: Reactive oxygen species and reactive nitrogen species. $^{5}$

Any free radical involving oxygen can be referred to as a ROS. Reactive oxygen species are highly reactive oxidant molecules that are generated endogenously through regular metabolic activity, lifestyle activity and diet. Free radicals and other reactive oxygen species (ROS) are the most biologically significant free radicals. ${ }^{6}$ They react with cellular components, causing oxidative damage to such critical cellular biomolecules as lipids, proteins and DNA. ${ }^{7}$ ROS have a very short life and are therefore not easy to detect. Most ROS have extremely short half-lives $\left(10^{-9}-10^{-6} \mathrm{~s}\right.$., they can cause substantial tissue damage by initiating free radical chain reactions. ${ }^{8}$ Reactive oxygen metabolites are short-lived and that proteases are either rapidly inactivated by circulating anti-proteinases or are released from PMNs as latent or inactive molecules. ${ }^{9}$ ROS are highly toxic, not only to the internalized microbial agent, but also to the extracellular structure and can induce lipid peroxidations (LPO) having effects on cells. Redundant production of LPO can result in oxidative stress and consequently, damage to cell integrity. ${ }^{10} \mathrm{An}$ early clue that such roles for ROS 
and RNS might be biologically important came from comparing the different patterns of antioxidant protection used by cells, membranes, and extracellular fluids. Briefly, cells use enzymes and other antioxidants to control ROS levels so that intracellular iron can signal within cells for synthesis of iron-containing proteins to achieve iron homeostasis. ${ }^{1}$

Oxygen is required for all mammalian energy needs. Oxygen is used to oxidize molecules rich in carbon and hydrogen (i.e., nutrients) to produce the different forms of energy needed for life. The reduction of molecular oxygen to water is accompanied by a large free energy release that can give rise to Free Radicals (FR) and/or Reactive Oxygen Species (ROS). ${ }^{11}$ Healthy adult rats will die within 72 hours if placed in an atmosphere of $100 \%$ oxygen, only, five times. The normal concentration at sea level. ${ }^{3}$ Oxygen may be considered a gaseous nutrient that cannot effectively be replaced by any other element. It is required for all mammalian energy needs. The evolution of efficient aerobic respiration allowed the development of complex multicellular organisms (aerobes) that use oxygen to oxidise (i.e., burn) fuels rich in carbon and hydrogen (i.e., nutrients) to produce the different forms of energy needed for life. However, the reduction of molecular oxygen to water is accompanied by a large free energy release that results in a great variety of chemical species, such as intermediates, depending upon environmental conditions. $^{12}$ Oxygen centred free radicals contain two unpaired electrons in the outer shell. When free radicals steal an electron from a surrounding compound or molecule a new free radical is formed in its place. ${ }^{13}$ The energetic benefit of aerobic metabolism is associated with the generation of ROS capable of damaging biologically relevant molecules such as DNA, proteins, carbohydrates, and lipids. The oxidants are major determinants of gene expression. Reactive oxygen intermediates have been implicated in the activation of a variety of kinases [such as the Src kinase family; protein kinase C; mitogen-activated protein kinase, MAPK; and receptor tyrosine kinases] and transcriptional factors, such as AP-1 and NF-kB. ${ }^{3}$
The most common ROS include: the superoxide anion $\left(\mathrm{O}^{2-}\right)$, the hydroxyl radical $\left(\mathrm{OH}^{-}\right)$, singlet oxygen $\left({ }^{1} \mathrm{O}_{2}\right)$, and hydrogen peroxide $\left(\mathrm{H}_{2} \mathrm{O}_{2}\right)$. Superoxide $\left(\mathrm{O}^{2-}\right)$, hydrogen peroxide $\left(\mathrm{H}_{2} \mathrm{O}_{2}\right)$, and hydroxyl radical $\left(\mathrm{OH}^{-}\right)$, which are mutagens produced by radiation, are also by-products of normal metabolism. ${ }^{14}$ The reduced oxygen metabolites formed by the NADPH oxidase system, including $\mathrm{O}^{2-}$ and $\mathrm{H}_{2} \mathrm{O}_{2}$, may be toxic to many microorganisms. In contrast to $\mathrm{O}^{2-}, \mathrm{H}_{2} \mathrm{O}_{2}$ is capable of freely diffusing across the membranes of the neutrophil plasmalemma, phagolysosome, or target organism. An important "lethal hit" is the damage of large, vital targets of limited copy number, such as chromosomal DNA. $^{15}$

In recent years, the term "reactive oxygen species" (ROS) has been adopted to include molecules such as hydrogen peroxide $\left(\mathrm{H}_{2} \mathrm{O}_{2}\right)$, hypochlorous acid (HOCI) and singlet oxygen $\left({ }^{1} \mathrm{O}_{2}\right)$, which though, not radical in nature, are capable of radical transformation in the extra and intracellular environments. Whilst most ROS have extremely short half-lives, they can cause substantial tissue damage by initiating free radical chain reactions. Therefore the body contains a number of protective Anti-Oxidant (AO) mechanisms, whose specific role is to remove harmful oxidants (ROS), as soon as they form, or to repair the damage caused by ROS invivo. $^{2}$

Superoxide anions are formed when oxygen $\left(\mathrm{O}_{2}\right)$ acquires an additional electron, leaving the molecule with only one unpaired electron. Within the mitochondria $\mathrm{O}^{2-}$ is continuously being formed. The rate of formation depends on the amount of oxygen flowing through the mitochondria at any given time. Hydroxyl radicals are short-lived, but are the most damaging radicals within the body. ${ }^{14}$ The superoxide radical anion appears to play a central role, since other reactive intermediates are formed from it. Superoxide is formed by one-electron reduction of oxygen mediated by enzymes such as NADPH oxidase or xanthine oxidase or by the respiratory chain. $^{16}$ Leucocytes are capable of producing superoxide at a rate of $5.8 \mathrm{nM} / 10^{6}$ cella/ $/ 15 \mathrm{~min} .{ }^{17}$ It is the homozygous SOD knockouts, however, that dramatically illustrate how toxic superoxide can be $S O D$ encodes the manganese-containing SOD that localizes to the mitochondrion, the site that is responsible by far for most of the cellular production 
of superoxide. SOD knockouts are born small, but alive, but they die within days of birth with a dilated cardiomyopathy. When first discovered as a biologic metabolite, it appeared that the superoxide radical was simply a noxious cytotoxic byproduct that served no good purpose. That view changed when Babior et al (1973) realized that the radical is an important player in our defense against invading microbes. It is now universally accepted that the production of superoxide radical by activated polymorphonuclear leukocytes ${ }^{3}$ and macrophages (and to a lesser extent eosinophils, lymphocytes and fibroblasts) are examples of cells that produce superoxide as an antibacterial agent. ${ }^{16}$ and other phagocytes is an essential component of their bactericidal armamentarium. In effect, superoxide serves as an extremely broad spectrum antibiotic. The neutrophil is also destroyed in the process, a Kamikaze mission, by its own artillery. In addition, surrounding healthy host cells may be injured or even killed in the crossfire. In effect, superoxide is a mediator of inflammation, and SODs display anti-inflammatory activity. It should be noted that our immune defense system tends to overreact to any challenge, as too timid a response may be fatal. The damage associated with the inflammatory process is the price we pay for a vigilant defense system. ${ }^{3}$

The hydroxyl radical can stimulate a classic free radical chain reaction known as lipid peroxidation. When the hydroxyl radical is generated close to membrane phospholipids, it attacks the lipid side chains to form radical intermediates called peroxyl radicals $\left(\mathrm{RO}_{2}\right)$, hydrogen peroxide and lipid hydroperoxides. Arachadonic acid is a preferential target for the hydroxyl radical. Mechanisms of preventing hydroxyl radical induced tissue damage include the binding of transition metal ions by the "preventative antioxidants" albumin, caeruloplasmin, haptoglobin, lactoferrin, and transferrin. Scavengers of the hydroxyl radical include vitamin $\mathrm{C}$, uric acid and thiols, such as reduced glutathione and cysteine. $^{16}$

Singlet oxygen, a high-energy and mutagenic form of oxygen, can be produced by transfer of energy from light, the respiratory burst from neutrophils, or lipid peroxidation. ${ }^{13}$ Singlet oxygen is molecular oxygen that has received an input of energy, which in turn allows a reversal of spin direction of one of the outer two unpaired electrons to occupy a high-energy state (anti-parallel spin). This creates a situation in which it is now possible for the outer two unpaired electrons to pair up, because the acquisition of two unpaired electrons with opposite spins is possible. Singlet oxygen, while not a true radical, is therefore highly reactive and capable of initiating lipid peroxidation from the side chains of polyunsaturated fatty acids. Eosinophils are a source of singlet oxygen but the reaction of ozone with biomolecules may also give rise to singlet oxygen. The "movement" of one of the unpaired electrons in a manner that alleviates the spin restriction yields singlet molecular oxygen or 102 (another ROS) generated in chemical as well as in biological systems, including medical conditions (e.g., the Porphyrias that are typical diseases in which excessive singlet oxygen is present; on the other hand, singlet oxygen is used in medicine in photodynamic therapy, e.g., to cure Herpes-Simplexinduced sores and psoriasis or to treat the jaundice of newborn babies. ${ }^{11}$ Removal of singlet oxygen is achieved by carotenoid pigments, which will absorb the energy of singlet oxygen and release heat. ${ }^{19}$

Hydrogen peroxide plays a key role in free radical biochemistry because, in the presence of transition metal ions, it can easily break down to produce the hydroxyl radical $(\mathrm{OH}-)$, one of the most reactive and damaging FR species. ${ }^{1}$

Another non-radical but highly reactive compound is hypochlorous acid (HOCI), produced during phagocytosis or as a result of myeloperoxidase activity on $\mathrm{H}_{2} \mathrm{O}_{2}$. $\mathrm{HOCI}$ is a powerful oxidizer of $-\mathrm{SH}$ groups on cell surfaces and can inhibit membrane transport systems as well as leading to chlorination of tyrosine residues. Hypochlorous acid is a powerful antibacterial agent and is also capable of causing oxidation of plasma membrane thiol $(\mathrm{SH})$ groups and disruption of certain protein functions, even at concentrations as low as $10-20 \mu \mathrm{m} .{ }^{16}$

Nitric oxide (NO,) provided the next clue as to how SOD might be playing a critical biological role. Although NO, is sluggish in its reactions with most biomolecules it is astoundingly reactive with free radicals, including superoxide. Overall, this high reactivity of $\mathrm{NO}$, with radicals may be beneficial in 
vivo, e.g. by scavenging peroxyl radicals and inhibiting lipid peroxidation. In some diseases, excessive production of NO will turn out to be important (e.g. septic shock perhaps), in others, one or more of the cytokines (e.g. TNF $\alpha$ in rheumatoid arthritis perhaps). The same is true of the reactive oxygen species. ${ }^{1}$

ROS have been associated with several diseases, playing a particular role in the female reproductive tract on ovaries, and even on embryos. ROS compounds are also implicated in the reproductive functions such as ovarian steroidogenesis, oocyte maturation, corpus luteal function and luteolysis, thus being related to female fertility. ${ }^{1}$

\section{OXIDATIVE STRESS}

Sies (1985) defined "oxidative stress" as "a disturbance in the pro-oxidant-antioxidant balance in favor of the former". ${ }^{4}$ Oxidative stress is caused by an imbalance between the production of reactive oxygen species and a biologic system's ability to readily detoxify the reactive intermediates or easily repair the resulting damage. $^{20}$ The connection between mild oxidative stress and cellular growth may date back to the primordial soup. When food is plentiful, metabolism is running at full speed, and there is sufficient energy to support cell division, the rate of superoxide production will also be high (at least in aerobic organisms), producing a state of mild oxidative stress. Conversely, when food supply nears exhaustion, the rate of [oxidant production within the cell would drop, possibly signaling insufficient energy production to support the cell's entry into a vulnerable period of replication. ${ }^{3}$

The number of oxidative hits to DNA per cell per day is about 10,000 in the human. DNA-repair enzymes efficiently remove most, but not all, of the lesions formed. Oxidative lesions and mutations also accumulate with age. For example, the somatic mutation frequency in human lymphocytes, of which the contribution of oxidative DNA lesions is unknown, is about 9 times greater in elderly people than in neonates. Cancer rates are high in a 2-yearold rat, but low in a 2-year-old human. One important factor in longevity appears to be basal metabolic rate, which is about 7 times higher in a rat than in a human and which could markedly affect the level of endogenous oxidants and other mutagens produced as by-products of metabolism. The level of oxidative
DNA damage appears to be roughly related to metabolic rate in a number of mammalian species (13). ${ }^{14}$ This oxidant stress is probably amplified by a continuing cycle of metabolic stress, tissue damage and cell death, leading to further free radical production. ${ }^{21}$ Stimulation of the oxidative burst is usually monitored by assays such as oxygen consumption, chemiluminescence, superoxide or hydrogen peroxide production as measured by the average increased activity of a large number of granulocytes. $^{22}$

Introduction of excess iron or copper ions into the extracellular environment has the potential to cause oxidative damage. Cell proliferation followed by apoptosis, and eventually necrosis are observed invitro when cells in culture are subjected to ever increasing conditions of oxidation. ${ }^{1}$

\section{BIOLOGICAL TARGETS FOR FR AND ROS}

There are five principal targets for FR and ROS in living cells:

(1) small organic biomolecules,

(2) proteins,

(3) nucleic acids,

(4) gene activation, and

(5) unsaturated fattyacids.

\section{(1) SMALL ORGANIC BIOMOLECULES}

Such compounds include the following: vitamins (ascorbic acid, carotenoids, cx-tocopherol, quinones), carbohydrates (glucose, ribose), amino acids (hystidine, tryptophane, cysteine, methionine), uric acid, cholesterol, and small soluble peptides like glutathione. The reaction of FR and ROS with vitamins $\mathrm{A}, \mathrm{C}$, or $\mathrm{E}$, quinones, glutathione, and uric acid usually terminates the radical reaction chain. Mucopolysaccharides such as hyaluronic acid can be depolymerized by FR/ROS. Carbohydrate affected by ROS leads to receptor alterations and reduced viscosity of synovial fluid. ${ }^{23}$

\section{PROTEINS}

The sensitivity of a protein to FR/ROS depends on its amino acid composition and on the accessibility of such species to its more susceptible amino acids. ${ }^{11}$ Several amino acids undergo direct oxidative modification, which may affect their physiological role, by interaction with FR and ROS. In two human diseases associated with premature aging, Werner syndrome and progeria, oxidized proteins increase at 
a much higher rate than is normal. ${ }^{14}$ The final molecular and biochemical consequences of the interaction of FR/ROS and proteins includes changes in conformation, enzymatic activity or binding as well as receptor inactivation, increased susceptibility to proteases, and changes in immunogenicity. ${ }^{11}$ Consequences of ROS on protein results in increased turnover, membrane damage and cell injury. ${ }^{23}$

\section{NUCLEIC ACIDS}

ROS alteration on DNA causes mutations leads to cell injury. ${ }^{23}$ The hydroperoxide of linoleic acid (1 3hydroperoxylinoleic acid) was found to cause guanine-site-specific double-stranded DNA breakage.

\section{GENE ACTIVATION}

FR/ROS have been capable of activating transcription and Encode transcription factors participating in the modulation of cell growth, differentiation, and development and can activate apoptosis, a "programmed" form of cell death. FR/ROS have been implicated in the regulation of mammalian transcription factors such as nuclear factor (NF)- $\kappa \mathrm{B}$ and activator protein-1 (AP-1) and of so-called "heat shock" (or stress protein) transcription factors (HSTF). ${ }^{23}$

\section{UNSATURATED FATTY ACID}

Free radical mediated lipid peroxidation leads to the formation of volatile breakdown products including aldehyde, ketone, alcohol etc and resulting in the irreversible damage to the placenta. Oxidized cholesterol derivatives are also implicated in human atherosclerosis and cardiovascular disease. Consequences of lipid peroxidation are membrane damage, LDL damage which leads to atherosclerosis. ${ }^{23}$ The key role of lipid peroxidation in causing rancidity in foods became increasingly apparent with the trend towards more pre-packaged "long life" food materials, kindling intense interest in the quantification of food oxidative deterioration and the development of protective antioxidants (of which the simplest is to pack food in vacuo or under nitrogen). The first toxin shown to act by a free radical mechanism, carbon tetrachloride $\left(\mathrm{CCl}_{4}\right)$, causes damage by stimulating lipid peroxidation, easily detected in animals, cells and microsomal fractions. Unlike $\mathrm{CCl} 4$ most toxins acting by free radical mechanisms do not primarily act via lipid peroxidation, and that oxidative damage to DNA and/or proteins is often more important as a primary cytotoxic mechanism than is lipid peroxidation. ${ }^{1}$ Lipid peroxidation gives rise to mutagenic lipid epoxides, lipid hydroperoxides, lipid alkoxyl and peroxyl radicals, and enals ( $\alpha, 4$-unsaturated aldehydes). ${ }^{14}$ The FR chain reaction consists of three essential steps-initiation, propagation, and termination and it propagates until two FR combine with each other to terminate the chain. ${ }^{11}$ Lycored might react with peroxy radicals, which are formed in propagation phase of lipid peroxidation to form carbon centered radical. The carbon centered radical reacts readily and reversibly with oxygen to form a new chain carry-ing peroxyl radicals, which are more highly stable forms than reactive oxygen species and thereby inhibiting lipid peroxidation. ${ }^{23}$

\section{EFFECTS OF ROS}

Free radicals and reactive oxygen species (ROS) are essential to many normal biologic processes. At low concentrations, these free radicals stimulate the growth of fibroblasts and epithelial cells in culture, but at higher concentrations it may result in tissue injury. ${ }^{24}$ A variety of biological processes, eg., antimicrobial defense, inflammation, carcinogenesis, radiation damage, photobiological effects, and aging, involve reactive oxygen species. ${ }^{25}$ Reactive oxygen species (ROS) play crucial roles in normal physiological processes including response to growth factors, the immune response, and apoptotic elimination of damaged cells but are also highly toxic and destructive when generated during the respiratory burst as it represents an important pathogenic mechanism for tissue damage and diseases associated with phagocytic insiltration. ${ }^{26}$ Excessive production of ROS resulting in oxidative stress has been implicated in the pathogenesis of many human diseases including periodontitis. ${ }^{18}$

Phagocytic cells destroy bacteria or virus infected cells with an oxidative burst of nitric oxide (NO), $\mathrm{O}_{2}$, $\mathrm{H}_{2} \mathrm{O}_{2}$, and $\mathrm{OC} 1$. Chronic infection by viruses, bacteria, or parasites results in a chronic phagocytic activity and consequent chronic inflammation, which is a major risk factor for cancer. Chronic infections are particularly prevalent in third-world countries, large endogenous oxidant load. The oxides of nitrogen $\left(\mathrm{NO}^{-}\right)$in cigarette smoke (about $1000 \mathrm{ppm}$ ) cause oxidation of macromolecules and deplete antioxidant levels. This is likely to contribute significantly to the pathology of smoking. Smoking is a risk factor for heart disease as well as a wide 
variety of cancers in addition to lung cancer. Leukocytes and other phagocytic cells combat bacteria, parasites, and virus-infected cells by destroying them with $\mathrm{NO}, \mathrm{O}_{2}, \mathrm{H}_{2} \mathrm{O}_{2}$, and $\mathrm{OC} 1$, a powerful oxidant mixture. These oxidants protect humans from immediate death from infection but cause oxidative damage to DNA and mutation, thereby contributing to the carcinogenic process. Helicobacter pylori bacteria, which infect the stomachs of over one-third of the world population, appear to be the major cause of stomach cancer, ulcers, and gastritis. ${ }^{14}$

Oxidative stress is now thought to make a significant contribution to all inflammatory diseases [arthritis, vasculitis, glomerulonephritis, lupus erythematosus, adult respiratory distress syndrome, ischemic diseases (heart disease, stroke, intestinal ischemia), hemochromatosis, acquired immunodeficiency syndrome (AIDS), emphysema, organ transplantation, gastric ulcers, hypertension and preeclampsia, neurologic diseases (multiple sclerosis, Alzheimer's disease, Parkinson disease, amyotrophic lateral sclerosis, muscular dystrophy), alcoholism, smoking-related diseases, and many others. ${ }^{3}$

The effects of ROS are generally negated by a complex system of antioxidants, such as glutathione, vitamin C. and vitamin E, as well as enzymes such as catalase, superoxide dismutase (SOD), and various peroxidases. ${ }^{20}$ Oxidative breakdown is also caused by free radical reactions; indeed, rapid bleaching by hydroxyl radicals is well known. ${ }^{27}$

\section{CONCLUSION}

Excess of everything is harmful such as no oxygen supply will prevent aerobes to live and similarly $100 \%$ oxygen can also lead to death to aerobes. Excessive ROS can cause chronic disease including cancer but inflammation cannot be controlled without ROS. Also excess or decrease of ROS can also affect fertility.

\section{REFERENCES}

1. Gutteridge JMC, Halliwell B. Free radicals and Antioxidants in the year 2000. A Historical look to the future. Ann. New York Academy of Sciences: 136-146.

2. Sharma A, Sharma S. Reactive Oxygen Species and Antioxidants in Periodontics: A Review. International Journal of Dental Clinics 2011;3(2):44-47.

3. McCord JM. The Evolution of Free Radicals and Oxidative Stress. Am. J Med. 2000;108:652-659.
4. da Silva FM, Marques A, Chaveiro A. Reactive Oxygen Species: A Double-Edged Sword in Reproduction. The Open Veterinary Science Journal, 2010;4:127-133.

5. Vaya J, Aviram M. Nutritional Antioxidants: Mechanisms of Action, Analyses of Activities and Medical Applications. Current Medicinal Chemistry-Immunology, Endocrine \& Metabolic. May 2001;1(1):99.

6. Shetti A, Keluskar V, Aggarwal A. Antioxidant: Enhancing oral and general health. Journal of Indian Academy of Oral Medicine and Radiology 2009;21(1):1-6.

7. Agarwal S, Rao AV. Tomato lycopene and its role in human health and chronic diseases. CMAJ 2000 Sept.;163(6):739744.

8. Chapple ILC. Reactive oxygen species and antioxidants in inflammatory diseases. J Clin Periodontol 1997;24:287-296.

9. Attman LC, Baker C, Eleckman P, Luchtel D, Oda D: Neutrophil-mediated damage to human gingival epithelial cells. J Periodont Res 1992;27:70-79.

10. Wei D, Zhang X-L, Wang Y-Z, Yang C-X, Chen G. Lipid peroxidation levels, total oxidant status and superoxide dismutase in serum, saliva and gingival crevicular fluid in chronic periodontitis patients before and after periodontal therapy. Australian Dental Journal 2010;55:70-78.

11. Battino M, Bullon P, Wilson M, Newman H. Oxidative Injury And Inflammatory Periodontal Diseases: The Challenge Of Anti-Oxidants To Free Radicals And Reactive Oxygen Species. Crit Rev Oral Biol Med 1999;10(4):458476.

12. Battino M, Ferreiro MS, Gallardo I, Newman HN, Bullon P. The antioxidant capacity of saliva. J Clin Periodontol 2002;29:189-194.

13. Chapple ILC, Milward MR, Ling-Mountford N, Weston P, Carter K, Askey K, Dallal GE, De Spirt S, Sies H, Patel D, Matthews JB. Adjunctive daily supplementation with encapsulated fruit, vegetable and berry juice powder concentrates and clinical periodontal outcomes: a doubleblind RCT. J Clin Periodontol 2012;39:62-72.

14. Ames BN, Shigenaga MK, Hagen TM. Oxidants, antioxidants, and the degenerative diseases of aging. Proc. Natl. Acad. Sci. September 1993;90:7915-7922.

15. Chapple ILC. Role of free radicals and antioxidants in the pathogenesis of the inflammatory periodontal diseases. J Clin Pathol: Mol Pathol 1996;49:247-255.

16. Stahl W, Sies H. Antioxidant Defense: Vitamin E and C and Carotenoids. Diabetes 1997; 46 (Suppl. 2): 14-18.

17. Bartold PM, Wiebkin OW, Thonard JC. The effect of oxygen-derived free radicals on gingival Proteoglycans and hyaluronic acid. J Periodontol Res 1984;19:390-400.

18. Kimura S, Yonemura $\mathrm{T}$, Kaya $\mathrm{H}$. Increased oxidative product formation by peripheral blood polvmorphonuclear leukocytes in human periodontai diseases. J Periodont Res 1993;28:197-203.

19. Chapple ILC, Mathews JB. The role of reactive oxygen and antioxidant species in periodontal tissue destruction. Perio 2000, 2007;43:160-232.

20. Chandra RV, Sandhya YP, Nagarajan S, Reddy BH, Naveen A, Murthy RV. Efficacy of lycopene as a locally delivered gel in the treatment of chronic periodontitis: Smokers vs nonsmokers. Quintessence Int. 2012;43:401-411.

21. Arora N, Avula H, Avula JK. The adjunctive use of systemic antioxidant therapy (lycopene) in nonsurgical 
treatment of chronic periodontitis: A short-term evaluation. Quintessence Int 2013;44:395-405.

22. Shrivastava LM. Concepts of Biochemistry. $1^{\text {st }}$ ed. New Delhi: CBS Publishers and Distributers; 2004.

23. Dahiya P, Kamal R, Gupta R, Bhardwaj R, Chaudhary K, Kaur S. Reactive oxygen species in periodontics. J Indian Soc Periodontol 2013;17(1):411-416.

24. Aggarwal S, Singh K, Nagpal M, Kaur K and Ahluwalia P. Studies on the effect of lycored supplementation (Lycopene) on lipid peroxidation and reduced glutathione in pregnancy induced hypertensive patients. Biomedical Research. 2009;20(1):51-54.
25. Mathur A, Mathur L, Manohar B, Mathur H, Shankarapillai R, Shetty N, Bhatia A. Antioxidant therapy as monotherapy or as an adjunct to treatment of periodontal diseases. $\mathrm{J}$ Indian Soc Periodontol 2013 Jan-Feb;17(1):21-24.

26. Schmidt AM, Weidman E, Lalla E, Yan SD, Hori O. Cao R, Brett JG, Lamster IB: Advanced glycation endproducts (AGEs) induce oxidant stress in the gingiva: a potential mechanism underlying accelerated periodontal disease associated with diabetes. J Periodont Res 1996;31:508-515.

27. Britton, G. Structure and properties of carotenoids in relation to function. FASEB J. 1995;9:1551-1558.

Source of Support: Nil, Confilict of Interst: None Declared 